\title{
Percepción del consumidor sobre los tipos de carne picada que se comercializan en Montevideo
}

\author{
Consumer's perception of different types \\ of ground meat sold in Montevideo
}

\begin{abstract}
Raggio, Laura ${ }^{(1)}$, Gámbaro, Adriana (2), Pagano, Teresa (1), Montesano, Ana (1), Garmendia, Juan (1). (1)Departamento de Alimentos, Escuela de Nutrición, Universidad de la República, UdelaR, Montevideo, Uruguay (2)Sección Evaluación Sensorial, Departamento de Ciencia y Tecnología de Alimentos, Facultad de Química, Universidad de la República, UdelaR, Montevideo, Uruguay.
\end{abstract}

Contacto: agambaro@fq.edu.uy

RECIBIDO: 30/05/2014 - APROBADO: 28/09/2014

\begin{abstract}
Resumen
La técnica de asociación de palabras fue utilizada para realizar un estudio preliminar de la percepción sobre los tipos de carnes picadas comercializadas en Montevideo. 60 consumidores recibieron cinco tarjetas con las palabras: Carne Picada Común, Magra, Premium, de Ternera y Especial y se les solicitó que escribieran las cuatro primeras imágenes, asociaciones, pensamientos o sentimientos que vinieran a su mente al leer cada tarjeta. Posteriormente, evaluaron cuán saludable consideraban que era cada tipo de carne picada. Las respuestas fueron agrupadas en 16 categorías. La Carne Picada Común fue percibida como un alimento con alto contenido de grasa y evaluada como poco saludable. Las carnes picadas Magra y Premium fueron percibidas como productos con bajo contenido graso, nutritivas, saludables y de precio elevado. La tipo Premium fue considerada, además, como un producto de calidad. La carne picada de Ternera fue percibida principalmente como un producto con una textura tierna, suave y blanda, mientras que en la carne picada Especial se encontraron contradicciones en las palabras utilizadas por los consumidores para su descripción (cara-económica, con poca grasa-con mucha grasa). Por medio de esta técnica se identificaron los atributos más relevantes en el proceso de selección y toma de decisiones de compra de este tipo de producto.

Palabras clave: Estudios con consumidores, carne picada, asociación de palabras.
\end{abstract}

\begin{abstract}
The word association technique was used to perform a preliminary of study consumer's perception of ground meat sold in Montevideo. A total of 60 consumers were presented with five cards with the words: Regular Ground Meat, Lean, Premium, Veal and Special and were asked to write the first four images, associations, thoughts or feelings that came to mind with each card. Afterwards, the participants assessed how healthy they considered each type of ground meat to be. The answers were grouped in 16 categories. Regular Ground Meat was perceived as a food product with high fat content, and assessed as not very healthy. Lean and Premium ground meats were perceived as having low fat content, being nutritious, healthy and expensive. The Premium ground meat was also considered a high quality product. Lean ground meat was perceived mainly as tender, soft and mushy, while for the Special ground meat contradictions in the choice of words were found (expensive-inexpensive, low fat-high fat). Through this technique the most relevant attributes in the selection process and decision making process were identified. Keywords: Consumer studies, ground meat, word association.
\end{abstract}

\section{Introducción}

Para el consumidor, un producto o servicio es más que la suma de sus atributos, implica también un componente emocional y un significado simbólico (McDonagh, et al., 2002). Las técnicas o pruebas proyectivas permiten una condición de libre interpretación y respuesta ante un estímulo ambiguo, buscando entender las motivaciones, sentimientos, creencias, actitudes y las razones profundas para seleccionar alternativas de respuestas dentro de un tópico específico (Donoghue, 2000; Vidal, et al., 2013). Si bien las técnicas proyectivas se crean y tienen aplicación en el ámbito psicológico, a partir de 1940 se empezaron a utilizar en los estudios de la conducta del consumidor e investigación de mercado. A partir de esta fecha las técnicas proyectivas hacen un aporte complementario a la información cuantitativa y se utilizan para entender el porqué de las percepciones, sentimientos y motivaciones de los consumidores; buscando el acceso a información que 
ellos almacenan en niveles más profundos y no conscientes, niveles a los cuales se puede acceder de una forma espontánea e incluso afectiva (Donoghue, 2000; Guerrero, et al., 2010), y permitiendo revelar información inaccesible que no está disponible cuando se trabaja con medios convencionales, directos y explícitos (Greenwald, et al., 1995).

En la literatura, las técnicas proyectivas aparecen clasificadas o subdivididas en cinco categorías (Vidal, et al., 2013; Catterall, 2000; Upadhyaya, 2012): técnicas de completar, técnicas de construcción, técnicas de ordenamiento, técnicas expresivas y técnicas de asociación. Dentro de esta última categoría se encuentra la técnica de asociación de palabras, según la cual se solicita que de una lista de palabras o frases se escriba lo que viene a la mente de la persona entrevistada, ya sean palabras, imágenes o pensamientos (Vidal, et al., 2013; Steinmann, 2009; Donoghue, 2000). De esta manera se asocia el estímulo presentado con la información dada.

Para los productos alimenticios, se considera que las primeras asociaciones que vienen a la mente de los consumidores son las más relevantes en el proceso de selección y la toma de decisiones de la compra de los productos alimenticios (Roininen, et al., 2006). La técnica de asociación de palabras ha sido aplicada recientemente para conocer la percepción de diversos alimentos como yogures (Ares, et al., 2008), alimentos tradicionales (Guerrero, et al., 2010), aceites (Gámbaro, et al., 2011), chocolate (Gámbaro y Ellis, 2012), ensaladas (Vidal, et al., 2013) y queso de cabra (Vieitez, et al., 2014), entre otros.

La carne picada es un producto de consumo popular para la población uruguaya, y se ha convertido en un componente cada vez más utilizado debido a su versatilidad como ingrediente. En Montevideo, el consumo aparente de carne picada común es 5 gramos/persona/día, mientras que el de carne picada especial o magra es de 15 gramos/persona/día. Los hogares de mayores ingresos adquieren la picada especial o magra y los de menores ingresos la carne picada común (Bove y Cerruti, 2008).

En el año 2010, el Instituto Nacional de Carnes (INAC) publicó un estudio sobre las preferencias de cortes en el que se observó que la carne picada pasó del tercer lugar de preferencia en 2005 al primer lugar en 2010 en las cuatro zonas en las que se dividió el país (INAC, 2010). El Decreto del Poder Ejecutivo 215/006 del 3 de julio de 2006 (Uruguay, 2006) reglamentó la composición y elaboración de la carne picada en Uruguay, nombrando tres tipos de carne picada que se pueden comercializar en el ámbito nacional: carnes picadas comunes, magras y extra magras. Solamente está reglamentado el contenido máximo de grasa total que debe tener la carne picada en forma genérica $(20 \%)$. En caso de que el comercio expendedor ofrezca para la venta carnes picadas magras o extra magras, deberá especificar el porcentaje de grasa total que contiene, no habiendo en la legislación límites superiores para los mismos.

A pesar de esta normativa, se encuentran en los comercios de Montevideo una disparidad de nombres con los que la carne picada se comercializa: Común, Magra, Especial, Super, Premium, Super especial, de Ternera, etcétera. Por ese motivo, el objetivo de este trabajo fue utilizar la técnica de asociación de palabras para realizar un estudio preliminar sobre la percepción de un grupo de consumidores uruguayos frente a los diferentes nombres con los que se comercializa la carne picada en los supermercados y carnicerías de la ciudad de Montevideo.

\section{Materiales y Métodos}

\section{Participantes}

El estudio se llevó a cabo en la ciudad de Montevideo, utilizando una muestra de conveniencia de 60 consumidores esporádicos y habituales de carne picada, reclutados en diversos lugares públicos (Mercado Agrícola de Montevideo, Facultad de Química y Hospital de Clínicas). La muestra se considera de conveniencia dado que los sujetos de la investigación fueron seleccionados para el estudio en función de la facilidad para el reclutamiento y de su interés en participar y no considerando las características que los hacen representativos de la población.

\section{Procedimiento}

Los tipos de carne picada seleccionados para este trabajo fueron los que se encontraron con mayor frecuencia en las principales cadenas de supermercados y carnicerías (Común, Magra, Premium, de Ternera y Especial). No se utilizaron todos los nombres de los tipos de carne picada que se encuentran en el mercado en este estudio por considerar que podía resultar agotador para el encuestado. Para obtener las asociaciones de palabras, se presentaron a los consumidores cinco tarjetas con las palabras: Carne Picada Común, Carne Picada Magra, Carne Picada Premium, Carne Picada de Ternera y Carne Picada Especial.

Las tarjetas fueron presentadas en orden aleatorio, diferente para cada consumidor. Se les solicitó que escribieran las cuatro primeras imágenes, asociaciones, pensamientos o sentimientos que vinieran a su mente al leer cada tarjeta.

Posteriormente, se les solicitó a los participantes evaluar cuán saludable consideraban que era cada tipo de carne picada, utilizando una escala de 9 puntos, con descripciones verbales en los extremos $(1=$ nada saludable a $9=$ muy saludable) (Ares y Gámbaro, 2007). La frecuencia de consumo de carne picada se evaluó con una escala de 9 puntos con descripciones verbales en los extremos $(1=$ nunca a $9=$ todos los días) (Gámbaro, et al., 2013). Al final, los participantes respondieron una pequeña encuesta donde se relevaron sus datos personales, los lugares de compra de carne picada y los tipos de carne picada que habitualmente compran.

\section{Análisis de datos}

Sobre los datos de cuán saludable consideraban cada tipo de carne picada se realizó un análisis de varianza y test de Tukey para determinar diferencias significativas $(\mathrm{p} \leq 0.05)$.

Las palabras asociadas a cada tipo de carne picada fueron analizadas cualitativamente. Se realizó un análisis inicial de los términos recurrentes, agrupando los términos con significado similar en categorías. El procedimiento se basó en la interpretación personal de los investigadores del significado de las palabras. El análisis se llevó a cabo de forma individual por dos de los investigadores y los resultados obtenidos fueron posteriormente discutidos en detalle entre todos los integrantes del equipo de investigación. Las categorías definitivas y su nombre fueron finalmente acordadas por consenso. La frecuencia de mención para cada categoría se determinó contando el número de participantes que utilizaron palabras similares para describir los conceptos incluidos dentro de 
cada categoría. Si el participante utilizaba más de una palabra dentro de la misma categoría, se consideraba como una sola mención. Por ejemplo, en la categoría «Alimentos», un mismo consumidor podría llegar a mencionar distintos tipos de alimentos (hamburguesas, tucos, etcétera), pero solo se consideró una mención en la categoría «Alimentos» para el cálculo de la frecuencia de mención. Una vez calculadas las frecuencias de mención, solo se consideraron las categorías mencionadas por más del $10 \%$ de los encuestados.

Para detectar diferencias en la percepción de cada tipo de carne picada, se realizó la prueba Q de Cochran para cada una de las categorías consideradas. La prueba Q de Cochran es una prueba estadística no paramétrica utilizada en el análisis de diseños de bloques al azar de dos vías para determinar si los tratamientos tienen efectos idénticos cuando la variable de respuesta es binaria.

Posteriormente se realizó un Análisis de Correspondencia para observar gráficamente la relación entre los tipos de carne picada y las categorías de palabras. El Análisis de Correspondencia es una técnica descriptiva/exploratoria diseñada para analizar tablas de contingencia de dos vías que muestran un cierto grado de correspondencia entre filas y columnas (Greenacre, 2010).

Los análisis estadísticos se realizaron utilizando el software XL -Stat 2012 (Addinsoft, NY).

\section{Resultados}

La muestra estuvo compuesta por un $78 \%$ de mujeres y $22 \%$ de hombres, y por un $49 \%$ de egresados universitarios, $36 \%$ de estudiantes de educación terciaria y $15 \%$ de individuos con menor nivel de estudios. La edad de los participantes estuvo comprendida entre 25 y 75 años (promedio $43 \pm 13$ años). Al ser una muestra de conveniencia, mostró un sesgo hacia las mujeres y hacia los individuos con mayor nivel de estudios, ya que son los grupos de consumidores más afines a contestar encuestas.

La frecuencia de consumo promedio de carne picada de la población encuestada fue de 4,0 (en escala de 9 puntos), con un $44,1 \%$ de consumo bajo (puntajes 1 a 3 ), un $47,5 \%$ de consumo medio (puntajes 4 a 6 ) y un $8,5 \%$ de consumo alto (puntajes 7 a 9). En cuanto al lugar de compra, el 77\% compra la carne picada en los supermercados y el $23 \%$ en carnicerías.

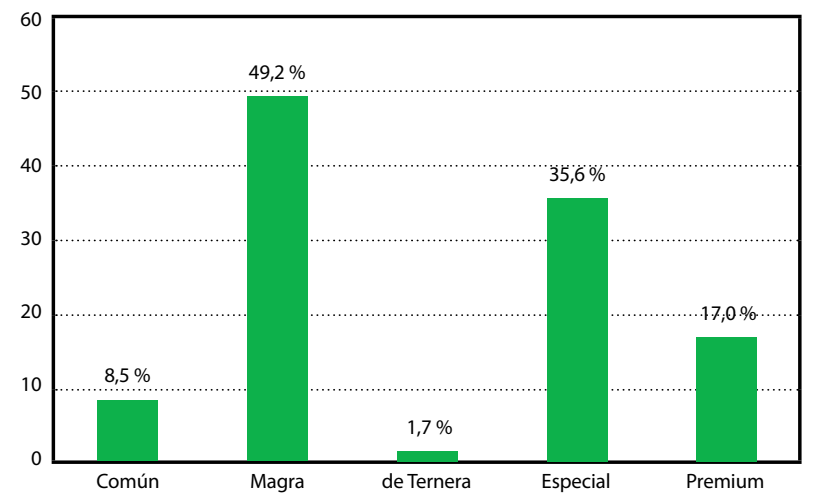

Gráfico 1. Porcentaje de los consumidores encuestados que consumen habitualmente cada tipo de carne picada.
En el Gráfico 1 se muestra el porcentaje de los consumidores encuestados que consumen habitualmente cada tipo de carne picada; la carne picada Magra y la Especial son las más consumidas.

De acuerdo al análisis de varianza realizado, se encontró diferencia altamente significativa $(\mathrm{p}<0,0001)$ en la percepción de cuán saludable es cada tipo de carne picada, como se observa en la Tabla 1. Las carnes picadas Premium y Magra fueron consideradas como las más saludables de todas. En segundo lugar aparecen las carnes picadas de Ternera y Especial, mientras que la Común fue percibida como muy poco saludable.

\begin{tabular}{|c|c|}
\hline Tipo de carne picada & $\begin{array}{c}\text { Percepción de saludable } \\
\text { (escala de 9 puntos) }\end{array}$ \\
\hline Premium & $7,1^{\mathrm{a}}$ \\
\hline Magra & $6,8^{\mathrm{a}}$ \\
de Ternera & $6,0^{\mathrm{b}}$ \\
Especial & $5,5^{\mathrm{b}}$ \\
Común & $3,8^{\mathrm{c}}$ \\
\hline
\end{tabular}

Tabla 1. Puntajes promedio de cuán saludable es percibido cada tipo de carne picada. Letras diferentes implican diferencia significativa según el test de Tukey $(\mathrm{p} \leq 0.05)$.

\section{Asociación de palabras}

Todos los participantes fueron capaces de proporcionar tres o cuatro asociaciones diferentes para cada tipo de carne picada. Las palabras fueron agrupadas en un número final de las siguientes 20 categorías: alimentos, agrado, apariencia, animal, calidad, cocción, consumo, conocimiento, equipamiento, dieta, desagrado, grasa, lugar de compra, ocasión, origen, olor, precio, sabor, salud y textura. De esas 20 categorías iniciales se eliminaron cuatro (conocimiento, equipamiento, origen y olor) que no alcanzaron un mínimo de un $10 \%$ de menciones en por lo menos uno de los tipos de carne picada.

En las Tablas 2 y 3 se observan las categorías resultantes, el porcentaje de menciones dentro de cada categoría y ejemplos de las palabras utilizadas por los participantes.

Según la prueba $\mathrm{Q}$ de Cochran, se encontraron diferencias significativas $(\mathrm{p} \leq 0.05)$ en 11 de las 16 categorías mencionadas por más del $10 \%$ de los participantes. El número mayor de menciones que recibieron los tipos de carne picada fueron relacionadas con los alimentos que la incluyen como ingrediente, con 131 menciones totales, seguida de la categoría grasa, con 105 menciones, y precio, con 93 menciones.

La carne picada Común fue percibida principalmente en relación con los alimentos que la incluyen como ingrediente (empanadas, pastel de carne, hamburguesas, tucos). Más de un 30\% de los consumidores hicieron mención a un alto contenido de grasa (grasosa, con grasa), mientras que las demás categorías recibieron un número más bajo de menciones.

La carne picada Magra fue percibida por más del $50 \%$ de los consumidores como un producto de bajo contenido graso (poca grasa, sin grasa) y por más del $47 \%$ de los consumidores como un producto nutritivo, saludable y sano. Más del 30\% 


\begin{tabular}{|c|c|c|c|c|c|c|}
\hline Categoría & Común & Magra & Premium & De Ternera & Especial & $P(Q$ de Cochran $)$ \\
\hline Alimentos $* * *$ & 83,1 & 37,3 & 28,8 & 28,8 & 44,1 & $<0,0001$ \\
\hline Agrado ns & 13,6 & 22,0 & 8,5 & 13,6 & 6,8 & 0,094 \\
\hline Apariencia ns & 23,7 & 20,3 & 27,1 & 23,7 & 25,4 & 0,885 \\
\hline Animal $* * *$ & 6,8 & 0,0 & 3,4 & 18,6 & 3,4 & 0,0002 \\
\hline Calidad $* * *$ & 11,9 & 16,9 & 47,5 & 13,6 & 25,4 & $<0,0001$ \\
\hline Cocción * & 0,0 & 10,2 & 3,4 & 1,7 & 3,4 & 0,027 \\
\hline Consumo* & 0,0 & 8,5 & 8,5 & 11,9 & 10,2 & 0,029 \\
\hline Dieta $* * *$ & 0,0 & 16,9 & 0,0 & 0,0 & 0,0 & $<0,0001$ \\
\hline Desagrado ns & 16,9 & 11,9 & 10,2 & 10,2 & 22,0 & 0,163 \\
\hline Grasa ${ }^{* *}$ & 32,2 & 50,8 & 37,3 & 16,9 & 40,7 & 0,001 \\
\hline Lugar de compra ns & 20,3 & 13,6 & 22,0 & 11,9 & 16,9 & 0,335 \\
\hline Ocasión * & 11,9 & 5,1 & 1,7 & 1,7 & 5,1 & 0,048 \\
\hline Precio $* * *$ & 13,6 & 32,5 & 50,8 & 18,6 & 42,4 & $<0,0001$ \\
\hline Sabor ns & 6,8 & 5,1 & 8,5 & 11,9 & 8,5 & 0,709 \\
\hline Salud $* * *$ & 0,0 & 47,5 & 25,4 & 11,9 & 8,5 & $<0,0001$ \\
\hline Textura $\star * \star$ & 1,7 & 3,4 & 3,4 & 44,1 & 5,1 & $<0,0001$ \\
\hline
\end{tabular}

${ }^{\star} P \leq 0.05,{ }^{* *} P \leq 0.01,{ }^{* * *} P \leq 0.001$, ns: Diferencia no significativa $(P>0.05)$ según la prueba $\mathrm{Q}$ de Cochran.

Tabla 2. Frecuencia (\%) de mención de cada categoría seleccionada.

de los consumidores hicieron mención al precio (cara, muy cara) y a los alimentos que la incluyen como ingrediente (canelones, empanadas, hamburguesas, pastel de carne, zapallitos rellenos).

Cerca del $50 \%$ de los consumidores percibieron la carne picada Premium como un producto de calidad (de exportación, excelente) y de precio elevado. Más del 37\% de los consumidores hicieron mención al contenido de grasa, percibiéndolo como un producto sin grasa o con menos grasa.

La carne picada de Ternera fue percibida principalmente como un producto con una textura tierna, suave y blanda. Se destaca también un número mayor de menciones en la categoría animal que en el resto de los tipos de carne picada, relacionadas con el propio nombre del producto. La carne picada Especial fue percibida mayormente en relación a su precio y contenido de grasa.

Estas relaciones entre los tipos de carne picada y las categorías obtenidas de la técnica Asociación de Palabras se reflejan en los resultados del Análisis de Correspondencia (Gráfico 2). Las dos primeras dimensiones explicaron el 79,8\% de la variabilidad de los datos experimentales. El factor 1 explicó el 44,5\% y el factor 2 el 31,8\% de la varianza.

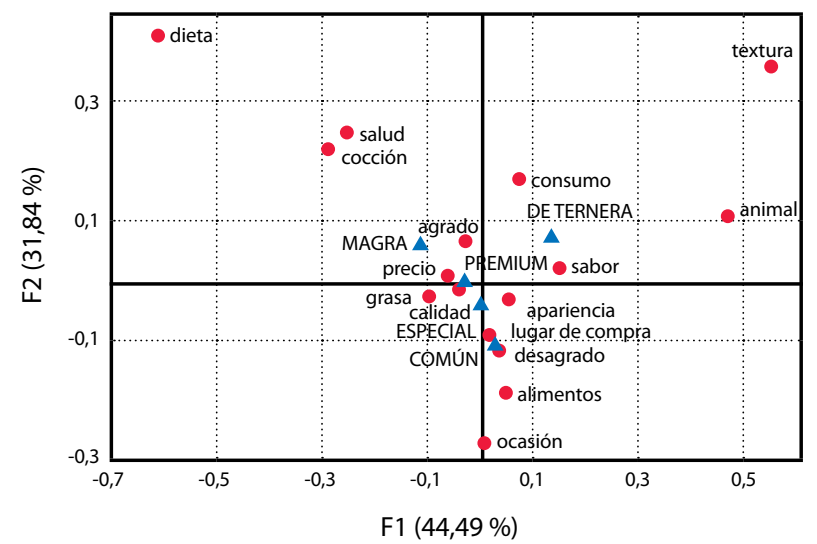

Gráfico 2. Análisis de Correspondencia para visualizar las asociaciones entre las categorías y los distintos tipos de carne picada. 


\begin{tabular}{|c|c|c|c|c|c|}
\hline Categoría & Común & Magra & Premium & De Ternera & Especial \\
\hline Alimentos & $\begin{array}{l}\text { Empanadas, } \\
\text { pastel de carne, } \\
\text { hamburguesas, } \\
\text { tucos }\end{array}$ & $\begin{array}{c}\text { Canelones, } \\
\text { empanadas, } \\
\text { hamburguesas, } \\
\text { pastel de carne, } \\
\text { zapallitos rellenos }\end{array}$ & $\begin{array}{l}\text { Comidas } \\
\text { especiales, } \\
\text { empanadas, } \\
\text { hamburguesas, } \\
\text { pan de carne }\end{array}$ & $\begin{array}{l}\text { Albóndigas, pan } \\
\text { de carne, guiso, } \\
\text { hamburguesas }\end{array}$ & $\begin{array}{c}\text { Canelones, tuco, } \\
\text { empanadas, } \\
\text { hamburguesas }\end{array}$ \\
\hline Agrado & Rica & Rica, me gusta & $\begin{array}{c}\text { Buena, me gusta, } \\
\text { la mejor }\end{array}$ & $\begin{array}{l}\text { Rica, apetitosa, } \\
\text { me gusta }\end{array}$ & Rica \\
\hline Apariencia & Roja & Roja, más roja & $\begin{array}{c}\text { Roja, } \\
\text { rojo intenso }\end{array}$ & Roja, rojo claro & Roja, no tan roja \\
\hline Animal & Vaca & ---- & Vaca & $\begin{array}{l}\text { Vaca joven, } \\
\text { ternera }\end{array}$ & Vaca, novillo \\
\hline Calidad & Calidad & $\begin{array}{l}\text { Calidad, fresca, } \\
\text { buen corte, } \\
\text { seleccionada }\end{array}$ & $\begin{array}{l}\text { Exportación, } \\
\text { calidad, excelente }\end{array}$ & $\begin{array}{c}\text { Calidad, fresca, } \\
\text { natural }\end{array}$ & $\begin{array}{c}\text { Calidad, } \\
\text { seleccionada }\end{array}$ \\
\hline Cocción & ---- & $\begin{array}{l}\text { Se apelotona, } \\
\text { se reduce }\end{array}$ & ---- & Rinde más & Reduce mucho \\
\hline Consumo & ---- & $\begin{array}{c}\text { No la consumo, no } \\
\text { la compro }\end{array}$ & $\begin{array}{l}\text { No compro, } \\
\text { no consumo }\end{array}$ & $\begin{array}{l}\text { No consumo, } \\
\text { nunca usé }\end{array}$ & $\begin{array}{l}\text { No consumo, } \\
\text { no compro }\end{array}$ \\
\hline Dieta & ---- & $\begin{array}{l}\text { Baja en calorías, } \\
\text { alimento dietético, } \\
\text { engorda menos }\end{array}$ & ---- & ---- & ---- \\
\hline Desagrado & $\begin{array}{l}\text { Desconfianza, } \\
\text { rechazo, } \\
\text { no me gusta }\end{array}$ & $\begin{array}{l}\text { No me gusta, } \\
\text { no me agrada }\end{array}$ & $\begin{array}{l}\text { Desagrado, } \\
\text { invento para } \\
\text { dieta, engaño }\end{array}$ & $\begin{array}{l}\text { Engaño, } \\
\text { no me gusta }\end{array}$ & $\begin{array}{c}\text { Engaño, no me } \\
\text { gusta, comida de } \\
\text { animal }\end{array}$ \\
\hline Grasa & Grasa, grasosa & $\begin{array}{l}\text { Sin grasa, poca } \\
\text { grasa, casi sin } \\
\text { grasa }\end{array}$ & $\begin{array}{c}\text { Sin grasa, menos } \\
\text { grasa }\end{array}$ & $\begin{array}{c}\text { Poca grasa, } \\
\text { grasosa, más } \\
\text { grasa }\end{array}$ & $\begin{array}{l}\text { Grasa, menos } \\
\text { grasa, más grasa }\end{array}$ \\
\hline $\begin{array}{l}\text { Lugar } \\
\text { de compra }\end{array}$ & $\begin{array}{l}\text { Carnicería, } \\
\text { supermercado }\end{array}$ & $\begin{array}{l}\text { Carnicería, } \\
\text { supermercado }\end{array}$ & $\begin{array}{l}\text { Carnicería, } \\
\text { supermercado }\end{array}$ & $\begin{array}{l}\text { Carnicería, } \\
\text { supermercado }\end{array}$ & $\begin{array}{l}\text { Carnicería, } \\
\text { supermercado }\end{array}$ \\
\hline Ocasión & Almuerzo & $\begin{array}{l}\text { Reunión, cena, } \\
\text { familia }\end{array}$ & Restaurante & Almuerzo & Familia, reunión \\
\hline Precio & Barata, económica & Cara, muy cara & Cara, más cara & Cara, costosa & $\begin{array}{l}\text { Cara, barata, } \\
\text { económica, } \\
\text { accesible }\end{array}$ \\
\hline Sabor & Sabrosa & Desabrida, sosa & $\begin{array}{l}\text { Sabrosa, } \\
\text { buen sabor }\end{array}$ & Sabrosa, gustosa & Sabrosa \\
\hline Salud & ---- & $\begin{array}{c}\text { Nutritiva, } \\
\text { saludable, sana }\end{array}$ & Saludable, sana & $\begin{array}{l}\text { Saludable, } \\
\text { nutritiva }\end{array}$ & Saludable \\
\hline Textura & Fibrosa & Seca & Tierna & $\begin{array}{l}\text { Tierna, suave, } \\
\text { blanda }\end{array}$ & Jugosa, suave \\
\hline
\end{tabular}

Tabla 3. Ejemplos de asociaciones individuales dentro de cada categoría.

\section{Discusión}

Los participantes del estudio mostraron una frecuencia de consumo de carne picada entre media y baja. La carne picada Magra y la Especial son los dos tipos más consumidos habitualmente. El alto consumo de carne picada Magra podría estar vinculado a las características de la población estudiada, con un alto porcentaje de mujeres e individuos con alto nivel de estudios, quienes podrían tender a consumir productos con menor contenido de grasa, lo cual coincide con lo reportado por Bove y Cerruti (2008). Los resultados sobre cuán saludable se percibe cada tipo de carne picada pueden sugerir que el principal motivo de compra de la carne picada Magra es la salud, mientras que en la carne picada Especial pueden influir otros factores. Si bien la carne picada Premium fue también considerada como muy saludable, no fue de las más 
consumidas por la población encuestada; en este tipo de carne picada pueden también influir otros factores que expliquen su frecuencia de consumo, por ejemplo que no se encuentre para la venta en todos los establecimientos comerciales.

Los resultados del análisis de los datos obtenidos con la técnica de asociación de palabras indican que los entrevistados percibieron en forma diferente cada tipo de carne picada. Las categorías alimentos, grasa y precio fueron las que recibieron mayor número de menciones, lo que indica la importancia que le da el consumidor encuestado a estos atributos, y son las asociaciones que vienen a su mente más frecuentemente con los distintos tipos de carne picada. La frecuencia de mención se ha asociado con la importancia con que un concepto en particular se encuentra en la mente de los encuestados (Guerrero, et al., 2010). A su vez, las categorías conocimiento, equipamiento, origen y olor no alcanzaron el mínimo del $10 \%$ de menciones en ninguno de los tipos de carne picada estudiados, lo cual además está indicando que estas asociaciones no están presentes en la mente del consumidor a la hora de comprar o consumir este producto.

Que la carne picada Común fuera percibida por más del $30 \%$ de los consumidores como un producto con alto contenido de grasa (grasosa, con grasa) puede explicar que haya sido el tipo de carne considerado como el menos saludable. Sin embargo, no recibió menciones dentro de la categoría saludable (ni positivas ni negativas), a diferencia de los demás tipos de carne picada que fueron consideradas saludables, sanas y nutritivas. Esto implica que en la mente del consumidor uruguayo no es relevante la salud a la hora de comprar la carne picada Común, aunque cuando se le pregunta directamente, la considera como poco saludable.

El elevado puntaje asignado respecto a cuán saludable consideran la carne picada Magra (Tabla 1) podría explicarse por el alto número de consumidores que la percibe como un producto de bajo contenido graso (poca grasa, sin grasa) y nutritivo. Aunque también se hizo mención al precio (cara, muy cara), fue la carne picada más consumida (Gráfico 1) por la población encuestada, lo que podría indicar que el concepto de salud prevalece a la hora de comprar este tipo de producto.

En el caso de la carne picada Premium, que un alto número de consumidores la percibieran como un producto sin grasa o con menos grasa, explica que haya sido considerado como muy saludable (Tabla 1). Comparando la percepción de la carne picada Premium con la Magra se observa en la primera un menor número de menciones respecto a su bajo contenido de grasa y un número menor de menciones en la categoría salud; el consumidor la percibe principalmente como un producto caro y de alta calidad. Es decir, en la mente del consumidor uruguayo el concepto de salud relacionado con la carne picada Premium es menos relevante que en el caso de la carne picada Magra, aunque cuando se le pregunta directamente la considera como muy saludable. Esto podría también explicar el menor consumo de este tipo de carne picada entre la población encuestada. Las demás asociaciones realizadas por los consumidores con la carne picada Premium no permiten explicar la percepción de la misma como un producto de alta calidad, por lo que esta percepción podría estar vinculada exclusivamente al nombre que lleva.

La carne picada de Ternera fue percibida principalmente como un producto con una textura adecuada, relacionada con el animal que da origen al nombre del producto, pero no aparecen menciones en un número importante en otras categorías. Este tipo de carne fue la menos consumida por la población encuestada (solamente un 1,7\% la consumen habitualmente), lo que podría llevar a que no está presente en la mente del consumidor y por esto recibió un menor número de menciones que el resto de los tipos de carnes picadas del estudio.

En el caso de la carne picada Especial se observan contradicciones en las palabras utilizadas por los consumidores dentro de las dos categorías más mencionadas (precio y contenido de grasa), ya que algunos participantes la consideraron como un producto caro y con poca grasa, mientras que otros la consideraron como un producto económico, accesible y con mucha grasa. Esto demuestra que existe una variabilidad en las carnes picadas comercializadas con el nombre «especial», lo cual lleva a que el consumidor no tenga una percepción definida sobre este producto.

En el Gráfico 2 se puede observar cómo los distintos tipos de carne picada se encuentran relacionados con las distintas categorías de la técnica de asociación de palabras. El factor 1 estuvo positivamente correlacionado con los términos textura y animal y negativamente correlacionado con los términos dieta, salud y cocción, permitiendo la diferenciación principalmente de la carne picada Magra de la de Ternera. El factor 2 correlacionó positivamente con los términos dieta, salud, cocción, textura y consumo, y negativamente con los términos alimentos y ocasión, permitiendo diferenciar las carnes picadas Magras, Premium y de Ternera de la Común.

\section{Conclusiones}

Por medio de la técnica de asociación de palabras se evaluó la percepción de los consumidores de diferentes tipos de carnes picadas, lo cual permitió identificar los atributos que son más relevantes en el proceso de selección y la toma de decisiones de la compra. Este trabajo debería ser complementado con un estudio con un mayor número de consumidores y más representativo de la población de Montevideo, y con un análisis del contenido graso y de otros factores de calidad de los distintos tipos de carnes picadas que se comercializan en nuestro mercado que permita investigar cuán real es la percepción que el consumidor tiene sobre estos productos.

\section{Referencias}

Ares, G., Gámbaro, A., 2007. Influence of gender, age and motives underlying food choice on perceived healthiness and willingness to try functional foods. En: Appetite, 49(1), pp.148-158.

Ares, G., Giménez, A., Gámbaro, A., 2008. Understanding consumers' perception of conventional and functional yogurts using word association and hard laddering. En: Food Quality and Preference, 19, pp.636-643.

Bove, I., Cerruti, F., 2008. Los alimentos y bebidas en los hogares: ¿Un factor de protección o de riesgo para la salud y el bienestar de los uruguayos? Encuesta Nacional de Gasto e Ingresos de los Hogares 2005-2006. Montevideo: Instituto Nacional de Estadística.

Catterall, M., Ibbotson, P., 2000. Using projective techniques in education research. En: British Educational Research Journal, 26(2), pp.245-256.

Donoghue, S., 2000. Projective techniques in consumer research. En: Journal of Family Ecology and Consumer Sciences, (28), pp.47-53. 
Gámbaro, A., Dauber, C., Ares, G., Ellis, A.C., 2011. Studying Uruguayan consumers' perception of vegetables oils using Word Association. En: Brazilian Journal of Food Technology, Special Issue 2011, pp.131-139.

Gámbaro, A., Ellis, A.C., 2012. Exploring consumer perceptions about the different types of chocolate. En: Brazilian Journal of Food Technology, 15(4), pp.317-324.

Gámbaro, A., Ellis, A.C., Prieto, V., 2013. Influence of subjective knowledge, objective knowledge and health consciousness on olive oil consumption - a case study. En: Food and Nutrition Science, (4), pp.445-453.

Greenacre, M., 2010. Correspondence analysis. En: International Encyclopedia of Education. 3ra. ed. New York: Elsevier. ISBN: 978-0-08-044893-0.

Greenwald, A. G., Banaji, M. R., 1995. Implicit social cognition: Attitudes, self-esteem, and stereotypes. En: Journal of Personality and Social Psychology, (102), pp.4-27.

Guerrero, L., Claret, A., Verbeke, W., Enderli, G., ZakowskaBiemans, S., Vanhonacker, F., Issanchou, S., Sajdakowska, M., Granli, B.S., Scalvedi, L., Contel, M., Hersleth, M., 2010. Perception of traditional food products in six European regions using free word association. En: Food Quality and Preferences, (21), pp.225-233.

INAC, 2010. Conociendo las preferencias del consumo de carnes en Uruguay [En línea]. Montevideo: INAC. [Consulta: 05/05/2014]. Disponible en: http://www.inac.gub.uy/ innovaportal/file/7365/1/conociendo_las_preferencias_ de_consumo.pdf
McDonagh, D., Bruseberg, A., Haslam., C., 2002. Visual product evaluation: exploring users' emotional relationships with products. En: Applied Ergonomics, (33), pp.231-240.

Roininen, K., Arvola, A., Lähteenmäki, L., 2006. Exploring consumer' perceptions of local food with two different qualitative techniques: Laddering and word association. En: Food Quality and Preference, (17), pp.20-30.

Steinmann, R. B., 2009. Projective techniques in consumer research. En: International Bulletin of Business Administration, (5), pp.37-45.

Upadhyaya, M., 2012. Projective Techniques for Brand Image Dimensionality and using various techniques to Investigate and Improve the Brand Personality. En: Polish Journal of Management Studies, 6, pp.89-100.

Uruguay. Decreto 215/006, del 3 de Julio de 2006. Diario Oficial, 7 de julio de 2006, p.20.

Vidal, L., Gastón, A., Giménez. A., 2013. Projective techniques to uncover consumer perception: Application of three methodologies to ready-to-eat salads. En: Food Quality and Preferences, 28, pp.1-7.

Vieitez, I., Gámbaro, A., Callejas, N., Miraballes, M., Irigaray, B., 2014. Consumer perception about goat cheese using Word Association technique. En: Journal of Food Science and Engineering, En prensa. 